\title{
ABBONAMENTO 1999
}

\section{EPIDEMOLOGIA \\ EPSICHIATRIA SOCIALE}

Avviso agli abbonati

A partire dal 1999 la Rivista diverrà trimestrale

e perciò ogni anno usciranno quattro fascioli (non più tre) più i supplementi

\section{ATTENZIONE}

A chi si abbonerà per il 1999

sarà inviato in omaggio il CD-ROM contenente tutte le annate complete dal 1992 al 1998

compresi i supplementi

con un sofisticato motore di ricerca

\section{RINNOVI SUBITO, L'ABBONAMENTO CONVIENE!! \\ PER INFORMAZIONI: N. VERDE 167-259620}




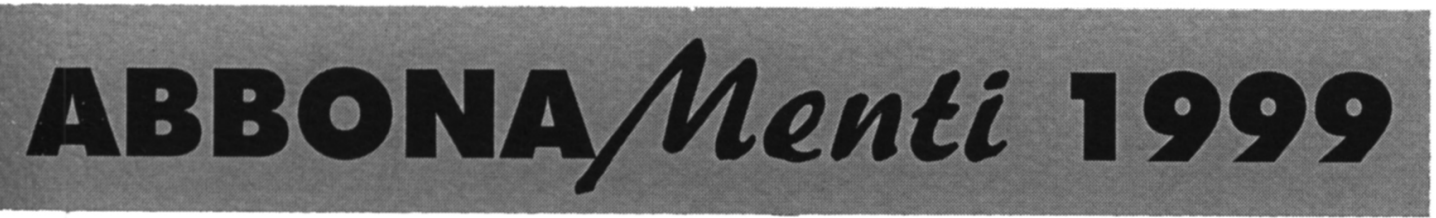

\section{ADOLESCENZA}

Quadrimestrale, diretto da N. Dazzi, S. Muscetta, A. Novelletto e A. Pazzagli Abbonamento individuale, lire 80.000 Istituti, enti, biblioteche, lire 120.000

Abbonamento cumulativo con Psicoanalisi, PPLI e Richard e Piggle, lire 250.000

\section{Ecolocia della Mente. Rivista} IMTERDISCIPLINARE PER LA COSTRUZIONE DI UN COMPORTAMENTO TERAPEUTICO

Semestrale, diretto da L. Cancrini Abbonamento individuale, lire 70.000 Istituti, enti, biblioteche, lire 100.000 Abbonamento cumulativo con Adolescenza, Psicoanalisi, PPLI e Richard e Piggle, lire 290.000

\section{Epidemiologia E Psichiatria Sociale}

Trimestrale, diretto da M. Tansella Abbonamento individuale, lire 100.000 Istituti, enti, biblioteche, lire 160.000 Abbonamento cumulativo con Rivista di Psichiatria, lire 190.000

\section{Rivista di Psichiatria}

Bimestrale, diretto da P. Pancheri e G.C. Reda. Redattore capo: M. Biondi Abbonamento individuale, lire 110.000 Istituti, enti, biblioteche, lire 170.000 Abbonamento cumulativo con Epidemiologia e Psichiatria Sociale, lire 190.000

\section{Prospettive Psicoanalitiche NEL LAVORO ISTITUZIONALE}

Quadrimestrale, diretto da M. Ammaniti, M. Bacigalupi e L.Carbone Tirelli Abbonamento individuale, lire 80.000 Istituti, enti, biblioteche, lire 120.000 Abbonamento cumulativo con Adolescenza, Psicoanalisi e Richard e Piggle, lire 250.000

\section{Giormale Italiano di Psico-oncologia}

Semestrale, diretto da G. Morasso

Organo della Società Italiana di Psicooncologia

Abbonamento individuale, lire 70.000

Istituti, enti, biblioteche, lire 90.000

\section{Psicoanalisi}

Semestrale, diretto da J. Amati Mehler Organo della Associazione Italiana di Psicoanalisi Abbonamento individuale, lire 70.000 Istituti, enti, biblioteche, lire 90.000

Abbonamento cumulativo con Adolescenza, PPLI e Richard e Piggle, lire 250.000

\section{Richard e Piggle. Studi psicoanalitici DEL BAMBINO E DELL'ADOLESCENTE}

Quadrimestrale, diretto da V. Bonaminio, L. Carbone Tirelli e G. Milana Abbonamento individuale, lire 80.000 Istituti, enti, biblioteche, lire 120.000

Abbonamento cumulativo con Adolescenza, Psicoanalisi e PPLI, lire 250.000

Per informazioni: Numero Verde 167-259620

Il Pensiero Scientifico Editore. Libri e riviste, nostri contemporanei 
In nessun altro campo dell' assistenza sanitaria si è verificato, negli ultimi vent' anni, un così profondo processo d'innovazione come nè campo della tutela della salute mentale. La crisi del vecchio modello culturale e operativo, basato sul manicomio, ha determinato assetti organizzativi dei servizi sempre più orientati verso pratiche territoriali, anche se molto ancora resta da fare in questa direzione. I processi di trasformazione della sanità, così come il governo del settore, devono fondarsi non solo sulla disponibilità delle risorse necessarie, ma anche sulla disponibilità di indicatori-descrittori adeguati. Per rispondere a questa esigenza, il Settore Medicina Sociale dell'Assessorato Salvaguardia e Cura della Salute della Regione Lazio ha istituito un Nucleo di Valutazione che ha prodotto questa monografia. Essa costituisce una prima fase elaborativa per la costruzione del sistema di indicatori per i Dipartimenti di Salute Mentale (DSM).

Epidemiologia e Psichiatria Sociale Monograph Supplements

1. Making Rational Mental Health Services (ed. M. Tansella)

2. Attività, Interventi e Strutture del DSM (F. Amaddeo et al)

3. Monitoraggio, Valutazione e Programmazione dei DSM (Progetto QUALI DSM, Regione Lazio) 